\title{
Prevalence of Hepatitis B and C Virus in Euthyroid Patients
}

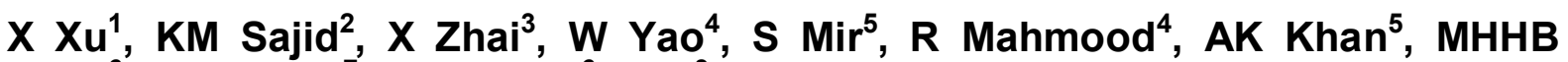 \\ Asad $^{6}, \mathrm{~K}_{\text {Farzana }}$, G Murtaza ${ }^{6}, \mathrm{~T} \mathrm{Li}^{3 *}$ \\ ${ }^{1}$ Zhengzhou Traditional Chinese Medicine Hospital, Zhengzhou, China, ${ }^{2}$ Multan Institute of Nuclear Medicines and Radiology, \\ Nishtar Hospital, Multan, Pakistan, ${ }^{3}$ Beijing University of Chinese Medicine, Beijing, ${ }^{4}$ China Development Bank, Beijing, China, \\ ${ }^{5}$ Department of Chemistry, ${ }^{6}$ Department of Pharmacy, COMSATS Institute of Information Technology 22060, Abbottabad, \\ ${ }^{7}$ Department of Pharmaceutical Sciences, Riphah International University, Lahore, Pakistan
}

*For correspondence: Email: gmdogar356@gmail.com; Tel: 0092-3142082826; Fax: 0092-992383441

\begin{abstract}
Purpose: To determine the prevalence of hepatic infections, viz, hepatitis $B$ (HBV) virus and hepatitis $C$ virus (HCV), in the euthyroid population of Southern Punjab Province of Pakistan.

Methods: A total of 120 euthyroid patients (36 male and 84 female) with a mean age of $30.7 \pm 0.09$ years) were included in this study. HBV and HCV, as well as the thyroid hormones, namely, triiodothyronine (T3), thyroxine (T4), and thyroid-stimulating hormone (TSH) were detected in the serum of the subjects using commercial kits.

Results: Out of 120 subjects 4 (3.3\%) were positive for HBsAg (hepatitis B surface antigen) and 18 (15 $\%)$ for anti-HCV. HCV infection was approximately 4 times higher than HBV infection $(p=0.00028)$. Prevalence of HBsAg in males (8\%) was significantly higher than in females $(1.2 \%, p=0.006345)$ whereas prevalence of HCV in males (16.7\%) was not significantly different from that in females (14.2 $\%, p=0.521303)$. HCV $(16.7 \%)$ was significantly higher than HBV infection $(8.33 \%, p=0.0102)$ in male sufferers. Similarly, among females patients, the incidence of HCV $(14.2 \%)$ was significantly higher $(p=0.000011)$ than HBV infection $(1.2 \%)$.

Conclusion: The prevalence of HCV is higher than that of HBV in the studied population; furthermore, this prevalence of HCV is higher than values reported in other studies. The high level of HCV infection in the studied population is of concern, indicating the need for preventive measures to be put in place.
\end{abstract}

Keywords: Hepatitis B, Hepatitis C, Prevalence, Euthyroid

Tropical Journal of Pharmaceutical Research is indexed by Science Citation Index (SciSearch), Scopus, International Pharmaceutical Abstract, Chemical Abstracts, Embase, Index Copernicus, EBSCO, African Index Medicus, JournalSeek, Journal Citation Reports/Science Edition, Directory of Open Access Journals (DOAJ), African Journal Online, Bioline International, Open-J-Gate and Pharmacy Abstracts

\section{INTRODUCTION}

Hepatitis-B is a severe hepatic infection caused by hepatitis B virus (HBV). An estimated 200,000,000 people have been infected by HBV, and more than 3.5 billion have chronic (longterm) liver infections with hepatitis B worldwide [1]. Hepatitis $C$ is also a severe disease affecting majorly the liver, produced by the hepatitis $C$ virus (HCV) [2]. The infection is usually asymptomatic but chronic infection can appear as a scar on the liver and ultimately to cirrhosis, which is normally apparent after numerous years. It is approximated that approximately $3 \%$ of the world's population is living with chronic hepatitis C [3].

About 3 - 4 million people are infected yearly, out of which $>350,000$ die annually from hepatitis Cassociated diseases [3]. Infection rates have risen considerably in the 20th century owing to a combination of injection drug users (IDU) and intravenous therapy as well as poorly sterilized medical apparatus [4]. Unscreened blood 
transfusion, unchecked reuse of syringes and needles, use of contaminated tools by barbers and dental surgeons and tattooing have made it one of the biggest health concerns. The disease is transmitted via body fluids such as blood, semen, saliva and vaginal secretions.

In Pakistan, where a majority of the people are below the poverty line, the incidence of hepatitis is very high. It is the frequent cause of death in the country's population. Sufferers are usually neglected by society. According to some rough estimates, the hepatitis-infected population is around 15 million [5]. Health professionals and officials often make conflicting statements on the incidence rate of Hepatitis- $B$ in Pakistan, with incidence rate ranging from 1.7 to $5.5 \%[6,7]$.

Since, cirrhosis is one of the etiologies of euthyroid sickness [6], this study was carried out to estimate the incidence of $\mathrm{HBV}$ and $\mathrm{HCV}$ infection in the euthyroid population of Southern Punjab Province of Pakistan and to suggest a strategy for extensive future population studies.

\section{EXPERIMENTAL}

\section{Subject}

A total of 139 patients (in series in records) referred to MINAR for thyroid function tests in December 2011 were investigated for HCV and HBV infection. The levels of these hormones in these patients and their history showed that 7 (5 $\%)$ patients were hypothyroid and $12(8.6 \%)$ were hyperthyroid. The patients were also checked by our clinicians to assess their health. A total of 120 euthyroid patients (males: 36 Females: 84) were therefore included in the study. The mean age of these persons was 30.7 \pm 9 years (range (18 - 52 years). The patients with thyroid disorders and disease were excluded. All the individuals belonged to Saraiki Wasaib (Southern Punjab), Pakistan.

\section{Sample collection}

Three milliters blood was collected from each patient. Serum was used for detection of HBV and HCV which was separated from clotted blood by centrifugation. Tests for thyroid hormones, namely, triiodothyronine (T3), thyroxine (T4), and thyroid-stimulating hormone (TSH) were advised. The consent of the patients to use their test samples and thyroid hormone values for our research was obtained. They were also requested to permit the test for HBV and $\mathrm{HCV}$ in their serum samples without at no cost to them. A consent form was used to secure the consent of each patient.

\section{Testing of blood samples}

Each sample was tested for HBV and HCV using commercially supplied kits of HBV (One Step Test Device, Standard Diagnostics, Korea) and HCV (One Step Test Device, Intac Products, China) for qualitative detection of HBV or HCV. The principle of the test is based on rapid chromatographic immunoassay. T3, T4 and TSH were measured by radioimmunoassay (RIA) methods.

\section{Data analysis}

Each sample was analyzed for $\mathrm{HBV} / \mathrm{HCV}$ and thyroid related hormone levels. The data obtained were grouped for comparison: Group-1: All subjects, Group-2: males, and Group-3: females. Different groups were compared using Chi square test. SPSS (version 17.0) was used for analysis of data with $p<0.05$ as the level of significance.

\section{Ethical approval}

The study was approved (approval no. 35/TD/MINAR/2011) by the Ethics Committee of Multan Institute of Nuclear Medicine and Radiotherapy (MINAR), Multan, Pakistan. Moreover, this study was conducted in accordance with the guidelines of Good Clinical Practice and Helsinki guidelines for human use in clinical practice [8].

\section{RESULTS}

The age-wise distribution of the studied euthyroid persons is displayed in Table 1.

Table 1: Age-wise distribution of euthyroid persons

\begin{tabular}{lccc}
\hline Age (years) & Male & Female & Total \\
\hline $10-20$ & 9 & 22 & 31 \\
$21-30$ & 10 & 27 & 37 \\
$31-40$ & 8 & 13 & 21 \\
$41-50$ & 6 & 13 & 19 \\
$51-60$ & 2 & 6 & 8 \\
$61-70$ & 1 & 3 & 4 \\
Total & 36 & 84 & 120 \\
\hline
\end{tabular}

The observed prevalence of HBV and HCV along with their T3, T4 and TSH levels is shown in Table 2. Out of 120 subjects, $4(3.3 \%)$ were positive for HBsAg and 18 (15\%) for anti-HCV. Thus, $3.3 \%$ prevalence of HBV and $18 \%$ prevalence of HCV were observed in the studied population. The data showed that prevalence of 
Table 2: $T_{3}, T_{4}$ and TSH levels (expressed as mean \pm SD) in euthyroid subjects infected with HBV or HCV

\begin{tabular}{|c|c|c|c|c|c|c|}
\hline & $\mathbf{N}$ & HBV+ & $\mathrm{HCV}+$ & Mean $T_{3}$ & Mean $\mathrm{T}_{4}$ & Mean TSH \\
\hline Males & 36 & $3(8.3 \%)$ & $6(16.7 \%)$ & $2.3 \pm 0.87$ & $103.9 \pm 19.2$ & $1.6 \pm 1.1$ \\
\hline Females & 84 & $1(1.2 \%)$ & $12(14.2 \%)$ & $2.1 \pm 1.0$ & $111.7 \pm 15.3$ & $1.34 \pm 1.1$ \\
\hline Overall & 120 & $4(3.3 \%)$ & $18(15 \%)$ & $2.1 \pm 0.9$ & $109 \pm 33.2$ & $1.2 \pm 0.98$ \\
\hline $\begin{array}{l}P \text {-values (Chi } \\
\text { square test) }\end{array}$ & \multicolumn{6}{|c|}{$\begin{array}{l}\text { HBsAg vs. HCV(All persons): }(p=0.00028) \\
\text { HBs males vs. females: }(p=0.006345) \\
\text { HCV males vs. females: }(p=0.521303) \\
\text { HBs males vs. HCV males: }(p=0.0102 \\
\text { HBs females vs. HCV females: }(p=0.000011)\end{array}$} \\
\hline
\end{tabular}

HCV infection was about 4 times higher than that of HBV infection $(p=0.00028)$. Prevalence of HBsAg in males $(8 \%)$ was significantly higher than that for females $(1.2 \%, p<0.006345)$ whereas prevalence of $\mathrm{HCV}$ in males $(16.7 \%)$ was not significantly different from that for females $(14.2 \%, p=0.521303)$. Comparison of males positive for HCV and HBV showed that the incidence of $\mathrm{HCV}$ infection (16.7\%) was significantly higher than for $\operatorname{HBV}(8.3 \%, p=$ 0.0102 ). Similarly comparison of females positive for HCV and HBV showed that the incidence of HCV infection (14.2\%) was significantly higher $(p=0.000011)$ than HBV $(1.2 \%)$.

\section{DISCUSSION}

Chronic liver disease (CLD) has emerged as a major public health issue not only in Pakistan but all over the world. Alcoholism, HBV and HCV are responsible for $75 \%$ of chronic liver diseases, such as cirrhosis $[7,8]$. Increase in the incidence of these viruses is promoted by repeated consumption of disposable items such as syringes by some physicians and quacks, particularly in the villages, which is claimed to be responsible for $69 \%$ of all cases in Pakistan $[9,10]$. In Pakistan, the incidence of anti-HCV antibodies has been estimated to be about $6 \%$ and that of hepatitis $B$ antigen to be approximately $1.7-5.5 \%[5,11]$. Chronic hepatitis $B$ and/or C may cause CLD and other pathological disorders such as portal hypertension, hepatic insufficiency, and hepatocellular cancer [11]. Although CLD is generally caused by viral hepatitis in Pakistan, this may be due to other comparatively less common and potentially treatable diseases, including autoimmune primary billiary cirrhosis and Wilson's disease [7]. Repeated studies on the prevalence of viral hepatitis could be helpful in controlling this infection.

The observed prevalence rate was $3.3 \%$ and 18 $\%$ for HBV and HCV, respectively. The prevalence of $\mathrm{HCV}$ is much higher when compared to prevalence of HBV and findings of international research. The high level of this infection (different from already reported figures) in our region should be noticed by health authorities for control measures. High prevalence of HBV in males compared to females also needs attention. The sources of exposure of the males to this infection may be different from females. This needs further studies. Prevalence of HCV is almost the same in both the sexes. However, in both groups, the prevalence of HCV is much higher than that of HBV. Further studies can therefore, be suggested to determine the reasons for increased prevalence of $\mathrm{HCV}$ in euthyroid patients.

\section{Limitations of the study}

The sample size of this study is small thus the outcome of this study may not represent the whole population. Moreover, people with normal thyroid function are not included as control group in this study.

\section{CONCLUSION}

Prevalence of HCV is higher than HBV in the studied population; furthermore the prevalence rate of HCV obtained is higher than literature values. The increased level of HCV infection in the population calls for concern and therefore preventive measures are recommended.

\section{ACKNOWLEDGEMENT}

This research was supported by Project of Beijing University of Chinese Medicine (2014JYB-JS078 hold by Tiangang Li) and Young Talents Project of Beijing (YETP0821 hold by Xing Zhai).

\section{REFERENCES}

1. Ullah H, Khan SA, Ali S, Karim S, Baseer A, Chohan O, Hassan SMF, Khan KM, Murtaza G. Evaluation of self-medication amongst university students in Abbottabad, Pakistan; prevalence, attitude and causes. Acta Pol Pharm-Drug Res 2013; 70(5): 919922

Trop J Pharm Res, October 2014; 13(10): 1705 
2. Ryan KJ, Ray CG. Sherris Medical Microbiology. Ed. forth, McGraw Hill, 2004; pp 551-552.

3. Duan J, Zheng C, Gao K, Hao M, Yang L, Guo D, Wu J, Tian Y, Song X, Liu J, Guo S, Murtaza G, Zheng M. Ultrasonography of lower limb vascular angiopathy and plaque formation in type 2 diabetes patients and finding its relevance to the carotid atherosclerotic formation. Pak J Med Sci 2014; 30(1): 1-5.

4. Alter MJ. Epidemiology of hepatitis $C$ virus infection. WJG 2007; 13: 2436-2441.

5. Ali, S.A., Donahue, R.M.J., Qureshi, H. and Vermund, S.H. Hepatitis $B$ and hepatitis $C$ in Pakistan: prevalence and risk factors. Int J Infect Dis 2009; 13: 9-19.

6. Taha A, S Azhar, Lone T, Murtaza G, Khan SA, Mumtaz A, Asad MHHB, Kousar R, Karim S, Tariq I, Hassan SSU, Hussain I. Iron deficiency anaemia in reproductive age women attending obstetrics and gynecology outpatient of university health centre in Al-Ahsa, Saudi Arabia. Afr J Tradit Complement Altern Med 2014; 11(2): 339-342.

7. Khalida K. Situation of hepatitis in Pakistan and preparation of diagnostic reagents for the detection of
HBsAg ELISA. Ph.D thesis, Department of Biological Sci., Quaid-e-Azam University, Islamabad, Pakistan, 1997.

8. World Medical Association Declaration of Helsinki. Ethical principles of medical research involving human subjects. $\quad$ http://www.wma.net/en/30 publications/10plicies/b3/index.html. Accessed September 3, 2008.

9. Jafri $W$, Jafri $N$, Yakoob J, Islam M, Tirmizi SFA, Jafar $T$, Akhtar S, Hamid S, Shah HA, Nizami SQ. Hepatitis B and $C$ : prevalence and risk factors associated with seropositivity among children in Karachi, Pakistan. BMC Infect Dis 2006; 6: 101.

10. Tong CYW, Khan R, Beeching NJ, Tariq WUZ, Hart CA, Ahmad N, Malik IA. The occurrence of hepatitis $B$ and $C$ viruses in Pakistani patients with chronic liver disease and hepatocellular carcinoma. Epidemiol Infect 1996; 117: 327-332.

11. Daudpota $A Q$, Soomro AWS. Prevalence of hepatitis $B$ and $C$ in surgical patients. Pak J Med Sci 2008; 24: 483-484. 